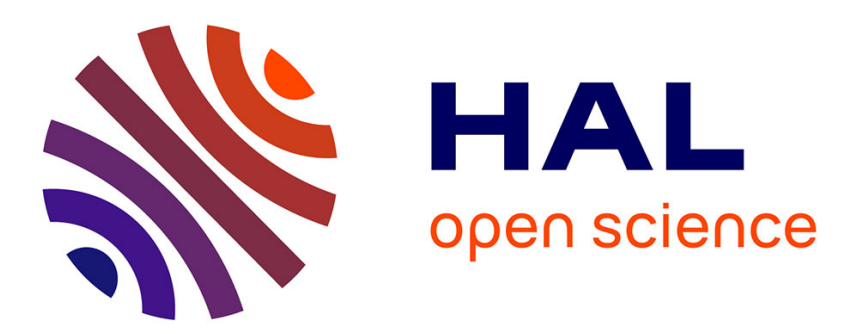

\title{
Coupling the first and second attenuation zones in seismic metasurface
}

Yi Zeng, Liyun Cao, Yifan Zhu, Yan-Feng Wang, Qiu-Jiao Du, Yue-Sheng Wang, B. Assouar

\section{- To cite this version:}

Yi Zeng, Liyun Cao, Yifan Zhu, Yan-Feng Wang, Qiu-Jiao Du, et al.. Coupling the first and second attenuation zones in seismic metasurface. Applied Physics Letters, 2021, 119 (1), pp.013501. $10.1063 / 5.0054606$. hal-03412453

\section{HAL Id: hal-03412453 \\ https://hal.science/hal-03412453}

Submitted on 3 Nov 2021

HAL is a multi-disciplinary open access archive for the deposit and dissemination of scientific research documents, whether they are published or not. The documents may come from teaching and research institutions in France or abroad, or from public or private research centers.
L'archive ouverte pluridisciplinaire HAL, est destinée au dépôt et à la diffusion de documents scientifiques de niveau recherche, publiés ou non, émanant des établissements d'enseignement et de recherche français ou étrangers, des laboratoires publics ou privés. 


\title{
Coupling the first and second attenuation zones in seismic metasurface
}

Yi Zeng ${ }^{1,2}$, Liyun $\mathrm{Cao}^{1}$, Yifan Zhu ${ }^{1}$, Yan-Feng Wang ${ }^{2}$, Qiu-Jiao Du ${ }^{3}$, Yue-Sheng Wang ${ }^{2}$, $4,{ }^{*}$ and Badreddine Assouar ${ }^{1, *}$

${ }^{1}$ Université de Lorraine, CNRS, Institut Jean Lamour, Nancy 54000, France

${ }^{2}$ Department of Mechanics, School of Mechanical Engineering, Tianjin University, Tianjin 300350, China

${ }^{3}$ School of Mathematics and Physics, China University of Geosciences, Wuhan 430074, China

${ }^{4}$ Department of Mechanics, Beijing Jiaotong University, Beijing 100044, China

* Authors to whom correspondence should be addressed: [Y.-S. Wang, yswang@tju.edu.cn; B. Assouar, badreddine.assouar@univ-lorraine.fr]

\begin{abstract}
Semi-infinite elastic metamaterials and metasurfaces on homogeneous elastic halfspaces have attracted significant attention in the past two decades as efficient artificial structures to control and mitigate surface waves. In this research, the first two attenuation zones of an elastic metasurface composed of different arrangements of pillars on a substrate are investigated. First, the lowest-frequency attenuation zone (LFAZ) is numerically investigated. Then, the rainbow trapping effect of the second attenuation zone (SAZ) is demonstrated by numerical simulations and experiments. The different characteristics of these two attenuation zones are brought together to connect lower bound of the SAZ and the upper bound of the LFAZ allowing designing a seismic
\end{abstract}


metasurface that only uses one kind of artificial structure on a half-space to attenuate Rayleigh waves in the frequency range extending from $5 \mathrm{~Hz}$ to $22 \mathrm{~Hz}$.

Keywords: Elastic metasurfaces; Seismic surface waves; Attenuation zone; Rainbow trapping effect 
In the past two decades, in the fields of applied physics, mechanics and civil engineering, the use of metamaterials and/or metasurfaces to control wave propagation has attracted great attention. ${ }^{1-13}$ Regarding surface waves, the lowest-frequency attenuation zone (LFAZ) of the elastic metasurfaces composed of an array of artificial structures on a substrate is the most appealing and interesting, since their working wavelengths are well above their characteristic size. ${ }^{11,14-17}$ In the LFAZ, a number of experiments ${ }^{15,18,19}$ and numerical studies ${ }^{11,12,14,16}$ have demonstrated that surface waves can be strongly attenuated because of the local resonance of the artificial structures on the substrate. It is worth mentioning that this conclusion is always valid whether the materials of the substrate and the artificial structures are the same or not. ${ }^{11,14-16}$ However, the width of the LFAZ is usually narrow. Therefore, the second attenuation zone (SAZ) whose frequency is slightly higher than the LFAZ attracted our attention. In the frequencies of the SAZ, Achaoui et al. ${ }^{15}$ experimentally demonstrated that surface waves are almost reflected in the elastic metasurface and the transmission is similar to the case of phononic crystals constituted of periodic holes in a substrate. In addition, the attenuation capacity of the elastic metasurfaces made of pillar-shaped artificial structure on a substrate significantly increases as the number of rows of the artificial structure increases. ${ }^{11,16}$ However, the frequency range of the SAZ is high. The problems in these two attenuation zones are the biggest bottleneck encountered in the current research on seismic metasurfaces (SMS). In 1999, phononic crystals composed of a periodic array of holes in a marble experimentally reported the existence of bandgap to attenuate elastic surface waves. ${ }^{20}$ It showed a possibility of application of the SMS on attenuation 
of seismic waves. In 2014, SMS composed of cylindrical holes arranged periodically in soil has been demonstrated in large-scale experiments to attenuate seismic waves at 50 Hz. ${ }^{21}$ However, the lower-frequency attenuation zones of the SMS require the local resonance mechanism ${ }^{22,23}$. Therefore, the SMS composed of periodic pillars on a substrate has attracted more attention, especially the LFAZ and the SAZ of the SMSs. These two attenuation zones are both located at low frequencies, i.e., the elastic metasurfaces are the subwavelength structures used to control surface waves. However, these two attenuation zones have both their own limitations. As mentioned earlier, although the LFAZ is at a lowest frequency, its relative bandwidth is usually very small. The SAZ has, on the other hand, a larger relative bandwidth than the LFAZ, but is located at a higher frequency range. Therefore, the shape of the pillars have been optimized to enlarge the relative bandwidth of the LFAZ and the SAZ. ${ }^{16,24}$ It also worth mentioning that hue and complex artificial structures have been introduced to achieve other attenuation zones in lower frequency range. ${ }^{24-26}$ Hence, the attenuation zone which can produce an ultra-low-frequency range of the LFAZ and a wide bandwidth of the SAZ at the same time, is worth to investigate.

On the other hand, the "rainbow trapping effect", i.e., using frequency selective structures with graded geometry or properties to filter waves in wide frequency ranges, has been observed in optics ${ }^{27}$ and acoustics ${ }^{28}$. Now, it has been extended to elastodynamics ${ }^{17,29-32}$. The broadband attenuation effect was experimentally demonstrated by using a scaled experimental model with an array of different resonant structures. ${ }^{17}$ In addition, by using the longitudinal-resonance bandgaps of pillars with 
varying heights, the proposed elastic metasurface not can only strongly attenuate surface waves in a wide frequency range, but the mode conversion of surface waves to bulk waves can also be found..$^{29,30}$ Recently, a kind of metabridge made of bridges of various sizes was proposed to protect bridges from earthquake efficiently by using destructive interferences and "rainbow trapping effect". ${ }^{31}$ However, the manufacturing process becomes complicated because of various resonance units or structures.

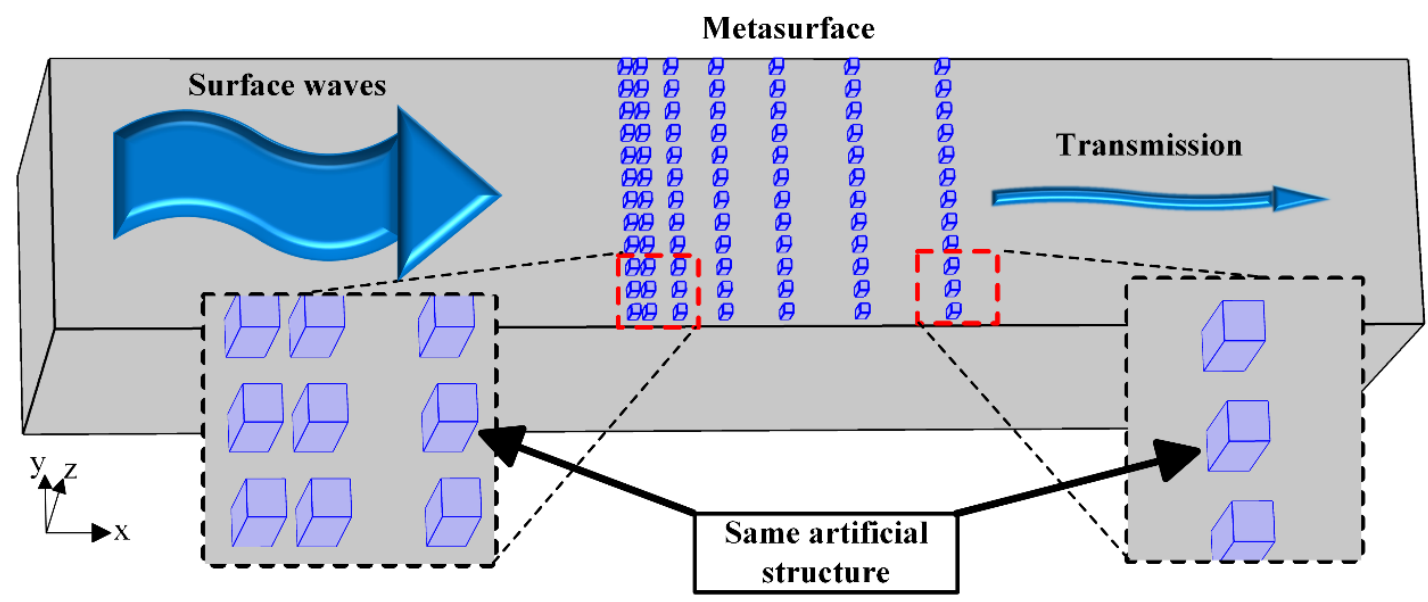

Figure 1 The schematic diagram of a kind of the elastic metasurface for surface waves. The metasurface is composed of just one kind of artificial structure but with different distances of every rows on a deep substrate.

In the present research, we propose alternative approaches to overcome these challenges. We report the properties of the LFAZ of a pillar-shaped elastic metasurface. Then, we experimentally demonstrate the "rainbow trapping effect" of the SAZ of an elastic metasurface composed of one kind of pillars with various lattice constants on a substrate. These two features of these two attenuation zones allow us to connect lower bound of the SAZ and the upper bound of the LFAZ, and design elastic metasurfaces using one kind of artificial structures to attenuate surface waves over a wide low-frequency range. 
Figure 1 shows the schematic diagram of the elastic metasurface. Finally, we design a seismic metasurface (SMS) just using one kind of artificial structure on a half-space to attenuate Rayleigh waves in a wide frequency range.

An elastic metasurface composed of steel pillars (width $w=0.3 \mathrm{~m}$, height $l=0.75 \mathrm{~m}$ ) periodically arranged with a lattice constant of $1.5 \mathrm{~m}$ on a soil substrate is taken as an example to investigate the properties of the LFAZ. The depth of the substrate is $H=$ $500 a$, which is much larger than two times of the wavelength $(2 \lambda)$ of the surface waves. The bottom surface of the unit cell is set as fixed boundary condition. The considered material parameters of steel and soil are same as the literature ${ }^{33,34}$.

Figure 2(a) shows the complex band structures of the elastic metasurface calculated by the PDE module of the COMSOL Multiphysics software. The dotted line of $c_{s}$ is the shear wave speed of the substrate material, i.e., the boundary of the sound cone. ${ }^{14,26}$ To draw all the surface modes in the band structure, the parameter $\xi$ is introduced, i.e.

$$
\xi=\int_{S(2 \lambda)}|\boldsymbol{u}| d s / \int_{S(H)}|\boldsymbol{u}| d s
$$

which is the ratio of the displacement $(\boldsymbol{u})$ in a depth of twice $(2 \lambda)$ of the Rayleigh wave wavelength to the entire depth $(H)$ of the substrate. ${ }^{11,35}$ The full complex band structure $(1>\xi>0)$ is shown on the left of the Fig. 2(a). Surface mode is defined as having parameter $\xi>0.9$. The complex band structure for surface modes $(\xi>0.9)$ is shown on the right of the Fig. 2(a). The first bandgap is found from 5.0 to 5.4 Hz. Although there is a band of surface modes from 5.0 to $5.2 \mathrm{~Hz}$, the surface waves are continuously attenuated as the distance traveled increases in this frequency range. Because the imaginary part of the wave vector of this band is positive, i.e., the wave mode of this 
band is evanescent ${ }^{36,37}$ with strong attenuation. The surface vibration mode, which falls before the $c_{s}$ line on this band, is shown in the far-right of Fig. 2(a). Most of the vibration exist in the substrate and decrease with increasing depth. The vibration mode of the pillars on substrate is similar as the one on the first band below $5.0 \mathrm{~Hz} .{ }^{11}$ It is worth noting that the curve of the imaginary part should start from the end of the real part at the high symmetry Brillouin points from 5 to $5.15 \mathrm{~Hz}$. However, the ideal results are difficult to calculate because the results are extremely dense at the junction.

The most interesting feature of the first bandgap is its immobility nature. The system composed of the substrate and an artificial structure is regarded as a spring-mass model. The resonance frequency of the spring-mass model is fixed if the substrate and the artificial structure are both not changed. So, the first bandgap due to the resonance of the spring-mass models is unchanged, even if the distribution of the artificial structures in the elastic metasurface varies. Figure 2(b) shows the upper and lower frequency bounds of the first bandgap when only the lattice constant of the elastic metasurface is changed. It is found that the first bandgap is absolutely unchanged due to the immobility of the lower frequency bound at $5.0 \mathrm{~Hz}$. The effects of other material parameters on the first bandgap are all calculated and shown in Supplementary Information (S1).

Figures 2(c) shows a part of the elastic metasurfaces composed by 30 steel pillars periodically arranged with a lattice constant of $1.5 \mathrm{~m}, 3 \mathrm{~m}$, and randomly arranged within $75 \mathrm{~m}$ on soil substrate, respectively. The geometric parameters of the steel pillars are consistent with those in Figs. 2(a). A perfectly matched layer (PML) with a thickness of $50 a$ is added around the substrate to prevent reflections. The transmission of 
Rayleigh waves in these three elastic metasurfaces are shown in Fig. 2(d). These three different arrangements have the same LFAZ around 5.0 Hz. This shows that the LFAZ is immobile with the changed distances between the pillars. Even if the same material is used for the pillars and the substrate, this phenomenon still exists, which has been experimentally demenstrated. ${ }^{19}$ It is worth noting that the strong attenuation of the surface waves in the SMS with the lattice constant of $3 \mathrm{~m}$ from 9 to $11 \mathrm{~Hz}$ is due to the existence of the second and third bandgaps.
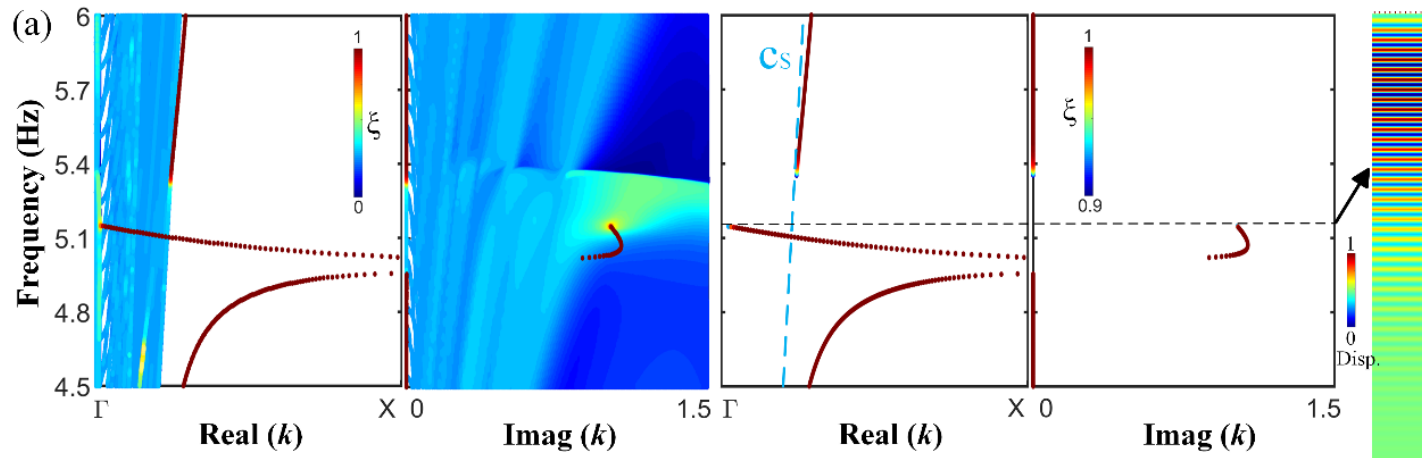

(b)
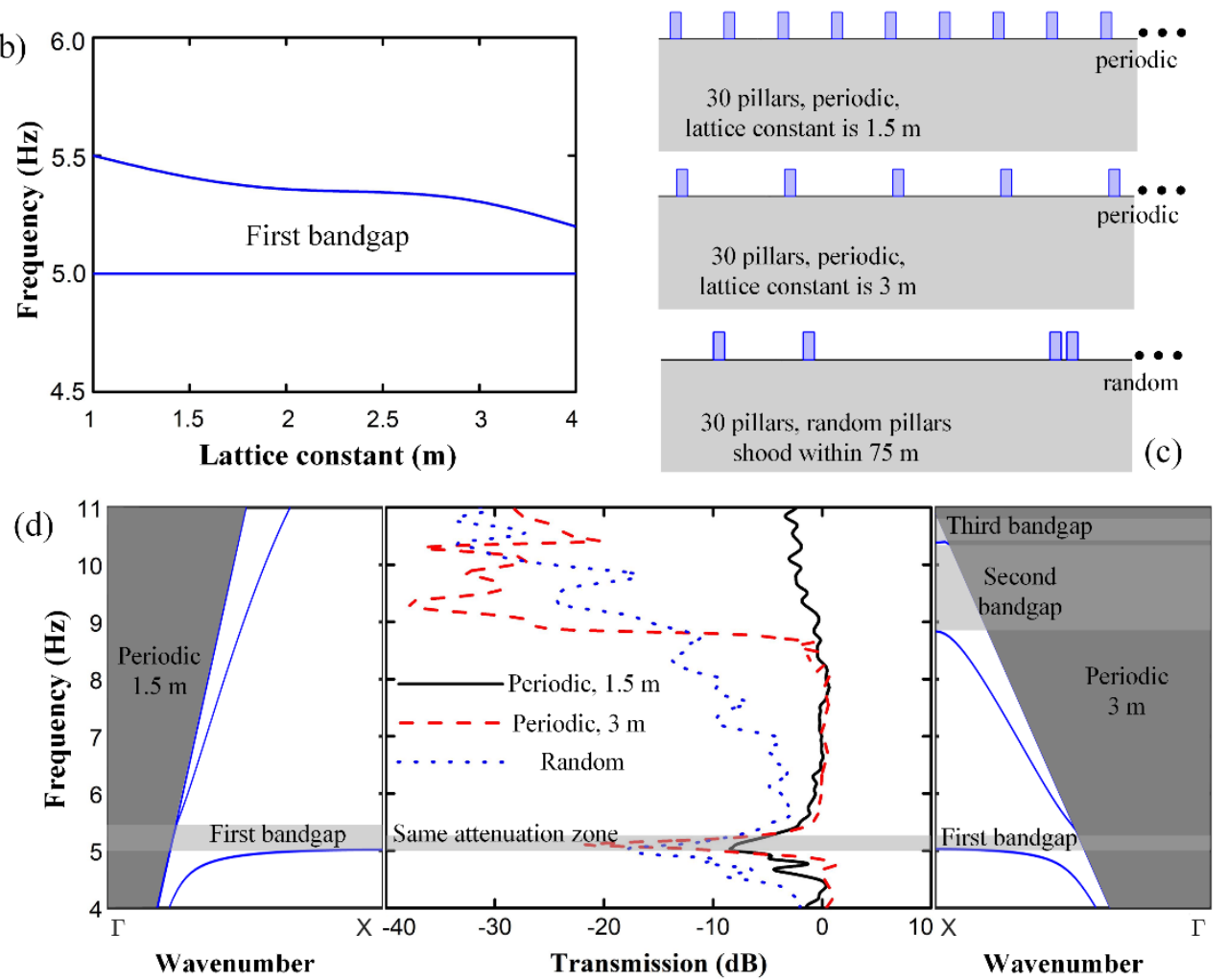

Figure 2 (a) The complex band structures of the elastic metasurface around the first bandgap. 
The left one is the full complex band structure $(1>\xi>0)$. The right one is the complex band structure for surface waves $(\xi>0.9)$. The far-right figure is the vibration mode of the SMS at $5.15 \mathrm{~Hz}$. (b) The upper and lower frequency bounds of the first bandgap with various lattice constants of the elastic metasurface. (c) Partial illustrations of two periodic arrangements of steel pillars with the lattice constants of $1.5 \mathrm{~m}, 3 \mathrm{~m}$ on a soil substrate and random arrangement of steel pillars on a soil substrate within $75 \mathrm{~m}$. (d) The transmission of Rayleigh waves in the three elastic metasurfaces. The far-left and far-right figures are the classic band structures of the SMSs with lattice constant $1.5 \mathrm{~m}$ and $3 \mathrm{~m}$, respectively. The same attenuation zone and bandgaps are marked by the light gray areas. The areas outside the dark gray areas are sound cones.

Table 1: The geometric parameters of the metasurface in Fig. 3.

\begin{tabular}{ccccc}
\hline$a$ & $b$ & $c$ & $h$ & $H$ \\
\hline Lattice constant & $15 \mathrm{~mm}$ & $6 \mathrm{~mm}$ & $7.5 \mathrm{~mm}$ & $300 a$ \\
\hline
\end{tabular}
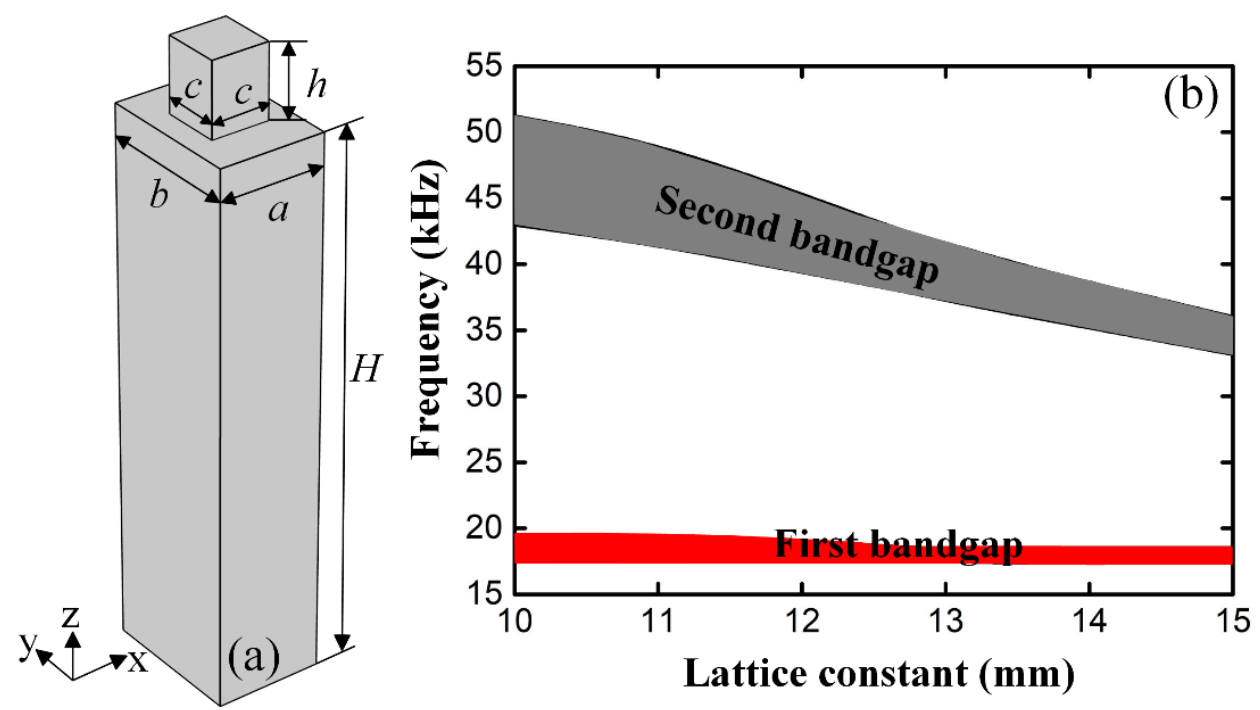

Figure 3 (a) The unit cell of the elastic metasurface for surface waves, (b) the effect of the lattice constant $a$ on the first and second bandgaps in the $Г \mathrm{X}$ direction of the elastic metasurface. 
The LFAZ generated by the elastic metasurfaces composed of an array of pillars on substrate is usually narrow. ${ }^{11,22,38,39}$ Therefore, to obtain a wider attenuation zone in the low-frequency range, the SAZ with a higher frequency than the LFAZ is investigated and discussed by using numerical simulations and experiments. Figure 3(a) shows the unit cell of the elastic metasurface whose band structure can be numerically simulated by using the solid module of COMSOL Multiphysics. In this work, the bandgaps only in the $\Gamma \mathrm{X}$ direction of the first irreducible Brillouin zone are investigated. The numerical computations are described in Refs. $\left.{ }^{14,26}\right]$. The material of the unit cell is PLA (its Young's modulus is $3.4398 \mathrm{GPa}$, density is $1086.3 \mathrm{~kg} / \mathrm{m}^{3}$, Poisson's ratio is 0.35$)^{40}$ and the geometric parameters are shown in Table 1. The characteristics of the first and second bandgaps are always valid and do not change, whether the materials of the substrate and the artificial structures are the same or not. ${ }^{11,14-16}$ Therefore, the proposed PLA models can accurately explore the basic properties of the bandgaps.

The effect of lattice constant $a$ on the upper and lower frequency bounds of the first and second bandgaps is shown in Fig. 3(b). The first bandgap is quite stable with various lattice constants, which is consistent with the previous results. However, the central frequency of the second bandgap keeps decreasing as the lattice constant increases. Therefore, this feature of the second bandgap is suitable to be used to obtain a wide attenuation zone at frequencies higher than the LFAZ through the "rainbow trapping effect"17,29,41,42.

Metasurface samples composed of three rows of PLA pillars with different lattice constants of $13.2,12.2$ and $11 \mathrm{~mm}$ on a PLA substrate have been considered for 
simulations and fabricated for experiments. Supplementary Information (S2) shows the detail information of the used geometric model in the simulation and experiment, and the setup of the experimental platform. From Fig. 3(b), it can be found that the SAZ of the considered sample extends from $37 \mathrm{kHz}$ to $50 \mathrm{kHz}$ due to the "rainbow trapping effect" of the second bandgap.
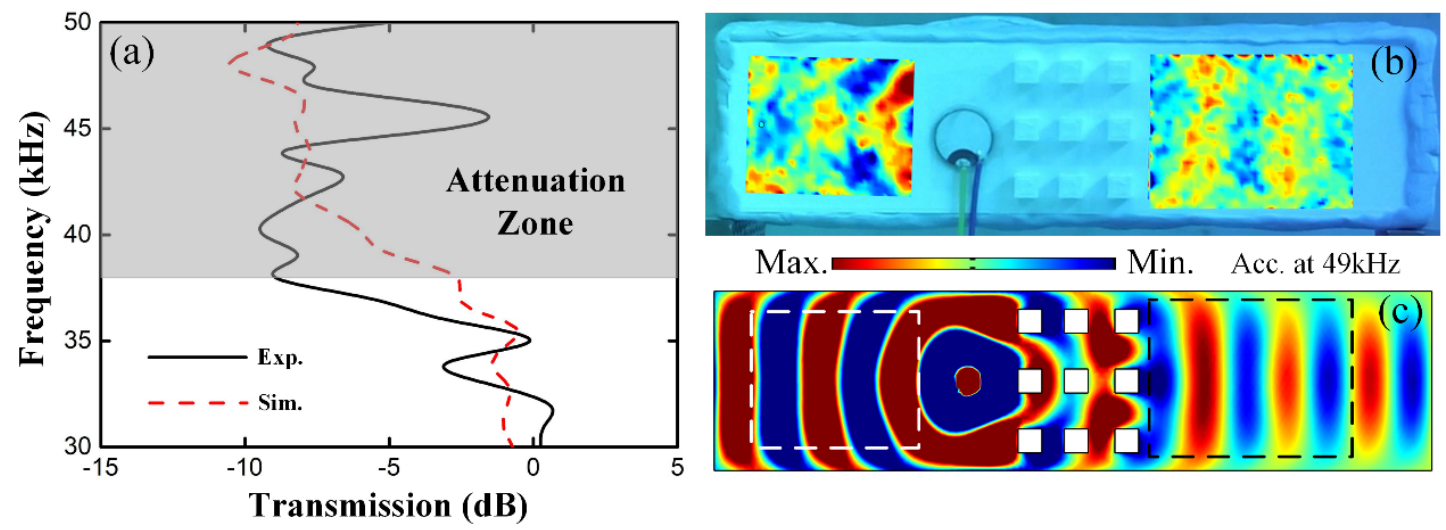

Figure 4 (a) The transmissions of surface waves on the prepared samples in simulations and experiments. Propagations of surface waves on the sample (b) in the experiment and (c) in the simulation at $49 \mathrm{kHz}$. The color indicates the acceleration field on the surface of samples.

Figure 4(a) illustrates the simulation and experimental measurements of the transmission of the surface waves through the considered metasurface sample. One can observe that in the range of $37-50 \mathrm{kHz}$, the elastic metasurface can significantly attenuate surface waves. We observe that there is a transmission peak at around $45 \mathrm{kHz}$. We do believe that the experimental transmission peak at around $45 \mathrm{kHz}$ is due to the imperfect absorption of the damping material covered the substrate for elastic waves. Figures 4 (b)-(c) show the out-of-plane acceleration fields on the surface of the samples in the experiment and simulation at $49 \mathrm{kHz}$, respectively. Limited to the number of rows of the pillars that compose the elastic metasurface, the attenuation effects do not seem 
obvious in these fields. However, when the number of rows of the pillars increases and more suitable lattice constants are chosen, better attenuation ability and a wider SAZ can be obtained. The elastic metasurface constituted of pillars with different distances on a substrate presents a wide SAZ to attenuate surface waves. It is worth noting that these pillars are all the same.

Indeed, when an artificial structure on the substrate is determined, the LFAZ is immobile even if the distances between the structures are changed. However, the SAZ whose frequency is higher than the LFAZ becomes wider when choosing the suitable distances between the structures due to the "rainbow trapping effect". Therefore, for a properly designed elastic metasurface, there is an opportunity to connect lower bound of the SAZ and the upper bound of the LFAZ. It is possible to propose an elastic metasurface with a wide attenuation zone from an ultra-low frequency for surface waves just using one kind of artificial structures on a substrate.

For instance, in this work, a kind of SMS with a wide attenuation zone for seismic Rayleigh waves is designed. Considering the wide range of the evanescent waves in the LFAZ of the seismic metasurface, the inverted T-shaped structure is chosen. ${ }^{43}$ As shown in Fig. 5(a), 15 identical inverted T-shaped steel structures are used. The geometric parameters are given in Table 2 . In the numerical simulation, the ideally perfect continuity is assumed between the inverted T-shaped structure and the substrate. The 15 steel structures are placed with appropriate distances. The distances between the symmetry axes of each steel structure in this research are 4.9, 4.7, 4.5, 4.3, 4.1, 3.9, 3.7, $3.5,3.3,3.1,2.8,2.4,2,1.65 \mathrm{~m}$. Of course, other suitable artificial structures, number 
of the structures and distances can also be selected. This paper only gives one possibility.
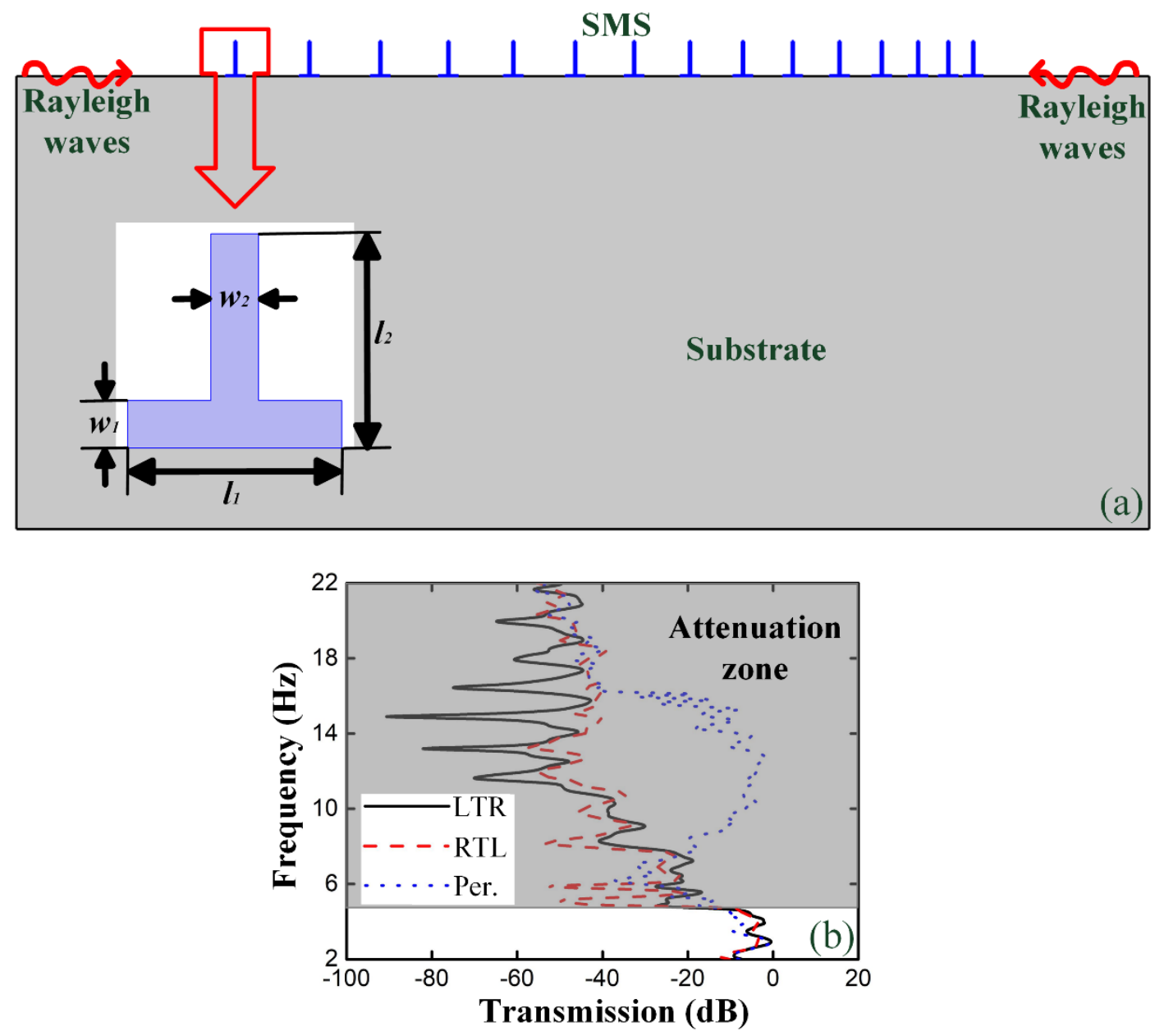

Figure 5 (a) Schematic picture of the finite system used for transmission calculations of the SMS, (b) the transmission of Rayleigh waves propagating in the SMS from different directions, and the transmission of Rayleigh waves propagating in a periodic arrangement of the 15 inverted T-shaped structures with a lattice constant of $1.5 \mathrm{~m}$. The gray area is the attenuation zone of the SMS. The LTR, RTL and Per. mean "from left to right", "from right to left" and "periodic", respectively.

Table 2: The geometric parameters of the inverted T-shaped structure

\begin{tabular}{cccc}
\hline$l_{1}$ & $w_{1}$ & $l_{2}$ & $w_{2}$ \\
\hline $1.35 \mathrm{~m}$ & $0.075 \mathrm{~m}$ & $2.25 \mathrm{~m}$ & $0.15 \mathrm{~m}$ \\
\hline
\end{tabular}


As shown in Fig. 5(b), when Rayleigh waves are incident from left to right (LTR) or from right to left (RTL), the SMS can all attenuate the energy well from 5.0 to $22.0 \mathrm{~Hz}$. The attenuation of the Rayleigh waves in the SMSs is complex because the attenuation zone includes the frequency ranges of the first and second bandgaps. There are two mechanisms of the attenuation of the Rayleigh waves in the seismic metasurface. The first one is the attenuation due to the local resonance of the artificial structures, while the second one is due to the conversion of the Rayleigh waves to bulk waves in the SMS. These two mechanisms of the attenuation of the Rayleigh waves in the SMS are shown in Supplementary Information (S3). Comparing with the SMS consisted of a periodic arrangement of inverted T-shaped steel structures with a lattice constant of 1.5 m on soil substrate, the "rainbow trapping effect" of the SMS works well to block the passband from 8.0 to $16.0 \mathrm{~Hz}$. It is worth noting that there are many kinds of threedimensional configurations coming from the two-dimensional one as shown in Fig. 5(a). The figure in Supplementary Information (S4) shows the schematic illustration of one possible three-dimensional configuration of the unit cell of the SMS.

In summary, the mechanical property of the LFAZ of the elastic metasurface made of pillars on a substrate has been investigated numerically. We found that the LFAZ is immobile with the changed distances between pillars. The "rainbow trapping effect" in the SAZ has then been demonstrated by numerical simulation and experiments. We have observed that the SAZ becomes wider when suitable distances between pillars are selected. Therefore, for a properly designed elastic metasurface, there is an opportunity to connect lower bound of the SAZ and the upper bound of the LFAZ. We then can 
obtain a wide attenuation zone from an ultra-low frequency for surface waves by just using one kind of artificial structures on a substrate. In the case of the seismic Rayleigh waves, these two characteristics of the LFAZ and the SAZ are used to design a seismic metasurface by just using one kind of artificial structure on a substrate. The proposed seismic metasurface can attenuation the Rayleigh waves from 5 to $22 \mathrm{~Hz}$. Different with the interplay of the local resonance and Bragg bandgaps ${ }^{44,45}$, the LFAZ and the SAZ of the SMS hardly interfere with each other and together create a common attenuation zone.

\section{Supplementary Information}

See the Supplementary Information for supporting content.

\section{Acknowledgement}

This work has been supported by la Région Grand Est, the Institut CARNOT ICEEL, and the National Natural Science Foundation of China (NNSFC) under Grant Nos. $11702017,11991031,11991032,12021002$ and 41974059. The first author is grateful for the support of China Scholarship Council (CSC Grant No. 202006250084).

\section{Data availability}

The data that support the findings of this study are available from the corresponding author upon reasonable request.

\section{References}

$1 \quad$ G. Wang, X. Wen, J. Wen, L. Shao, and Y. Liu, Physical review letters 93, 154302 (2004). https://doi.org/10.1103/PhysRevLett.93.154302.

G. Wang, J. Wen, Y. Liu, and X. Wen, Physical review B 69, 184302 (2004). https://doi.org/10.1103/PhysRevB.69.184302.

A. Khelif, B. Aoubiza, S. Mohammadi, A. Adibi, and V. Laude, Physical Review E 74, 046610 (2006). https://doi.org/10.1103/PhysRevE.74.046610.

$4 \quad$ Y. Pennec, J. O. Vasseur, B. Djafari-Rouhani, L. Dobrzyński, and P. A. Deymier, Surface Science Reports 65, 229 (2010). https://doi.org/10.1016/j.surfrep.2010.08.002. 
X.-Z. Zhou, Y.-S. Wang, and C. Zhang, Journal of Applied Physics 106, 014903 (2009). https://doi.org/10.1063/1.3159644.

L. Cao, Z. Yang, Y. Xu, S.-W. Fan, Y. Zhu, Z. Chen, Y. Li, and B. Assouar, Journal of the

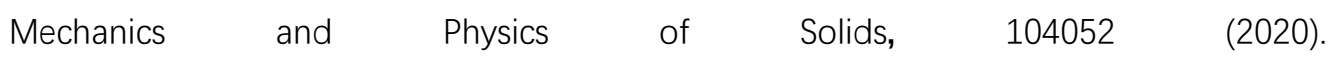
https://doi.org/10.1016/j.jmps.2020.104052.

J. Zhou, X. Zhang, and Y. Fang, Composite Structures 176, 1005 (2017). https://doi.org/10.1016/j.compstruct.2017.06.050.

X. Pu, A. Palermo, Z. Cheng, Z. Shi, and A. Marzani, International Journal of Engineering Science 154, 103347 (2020). https://doi.org/10.1016/j.ijengsci.2020.103347.

D. Colquitt, A. Colombi, R. Craster, P. Roux, and S. Guenneau, Journal of the Mechanics and Physics of Solids 99, 379 (2017). https://doi.org/10.1016/j.jmps.2016.12.004.

A. Diatta, Y. Achaoui, S. Brûlé, S. Enoch, and S. Guenneau, AlP Advances 6, 121707 (2016). https://doi.org/10.1063/1.4972280.

B. Graczykowski, F. Alzina, J. Gomis-Bresco, and C. Sotomayor Torres, Journal of Applied Physics 119, 025308 (2016). https://doi.org/10.1063/1.4939825.

A. Khelif, Y. Achaoui, S. Benchabane, V. Laude, and B. Aoubiza, Physical Review B 81, 214303 (2010). https://doi.org/10.1103/PhysRevB.81.214303.

Z. Liu, H.-W. Dong, and G.-L. Yu, Structural and Multidisciplinary Optimization 63, 463 (2021). https://doi.org/10.1007/s00158-020-02703-3.

M. Badreddine Assouar and M. Oudich, Applied Physics Letters 99, 123505 (2011). https://doi.org/10.1063/1.3626853.

Y. Achaoui, A. Khelif, S. Benchabane, L. Robert, and V. Laude, Physical Review B 83, 104201 (2011). https://doi.org/10.1103/PhysRevB.83.104201.

Y. Zeng, Y. Xu, K. Deng, Z. Zeng, H. Yang, M. Muzamil, and Q. Du, Journal of Applied Physics 123, 214901 (2018). https://doi.org/10.1063/1.5021299.

S. Krödel, N. Thomé, and C. Daraio, Extreme Mechanics Letters 4, 111 (2015). https://doi.org/10.1016/j.eml.2015.05.004.

S. Benchabane, R. Salut, O. Gaiffe, V. Soumann, M. Addouche, V. Laude, and A. Khelif, $\begin{array}{lllll}\text { Physical Review } & \text { Applied } & \text { 8, } & 034016 & \text { (2017). }\end{array}$ https://doi.org/10.1103/PhysRevApplied.8.034016.

Y. Achaoui, V. Laude, S. Benchabane, and A. Khelif, Journal of Applied Physics 114, 104503 (2013). https://doi.org/10.1063/1.4820928.

F. Meseguer, M. Holgado, D. Caballero, N. Benaches, J. Sánchez-Dehesa, C. López, and J. Llinares, Physical Review B 59, 12169 (1999). https://doi.org/10.1103/PhysRevB.59.12169. S. Brûlé, E. Javelaud, S. Enoch, and S. Guenneau, Physical Review Letters 112, 133901 (2014). https://doi.org/10.1103/PhysRevLett.112.133901.

Z. Liu, X. Zhang, Y. Mao, Y. Zhu, Z. Yang, C. Chan, and P. Sheng, Science 289, 1734 (2000). https://doi.org/10.1126/science.289.5485.1734.

P. Sheng, X. X. Zhang, Z. Liu, and C. T. Chan, Physica B: Condensed Matter 338, 201 (2003). https://doi.org/10.1016/s0921-4526(03)00487-3.

C. Lim and J. Reddy, Engineering Structures 188, 440 (2019). https://doi.org/10.1016/j.engstruct.2019.03.046.

Q. Du, Y. Zeng, Y. Xu, H. Yang, and Z. Zeng, Journal of Physics D: Applied Physics 51, 105104 (2018). https://doi.org/10.1088/1361-6463/aaaac0. 
Y. Zeng, Y. Xu, H. Yang, M. Muzamil, R. Xu, K. Deng, P. Peng, and Q. Du, International Journal of Solids and Structures 185-186, $334 \quad$ (2020). https://doi.org/10.1016/j.ijsolstr.2019.08.032.

K. L. Tsakmakidis, A. D. Boardman, and O. Hess, Nature 450, 397 (2007). https://doi.org/10.1038/nature06285.

V. Romero-García, R. Picó, A. Cebrecos, V. J. Sánchez-Morcillo, and K. Staliunas, Applied Physics Letters 102, 091906 (2013). https://doi.org/10.1063/1.4793575.

A. Colombi, D. Colquitt, P. Roux, S. Guenneau, and R. V. Craster, Scientific Reports 6, 27717 (2016). https://doi.org/10.1038/srep27717.

A. Colombi, V. Ageeva, R. J. Smith, A. Clare, R. Patel, M. Clark, D. Colquitt, P. Roux, S. Guenneau, and R. V. Craster, Scientific Reports 7, 1 (2017). https://doi.org/10.1038/s41598-017-07151-6.

W. Liu, G. H. Yoon, B. Yi, Y. Yang, and Y. Chen, Extreme Mechanics Letters 41, 101018 (2020). https://doi.org/10.1016/j.eml.2020.101018.

S.-M. Yuan, T.-X. Ma, A.-L. Chen, and Y.-S. Wang, AIP Advances 8, 035026 (2018). https://doi.org/10.1063/1.5011194.

M. Miniaci, A. Krushynska, F. Bosia, and N. M. Pugno, New Journal of Physics 18, 083041 (2016). https://doi.org/10.1088/1367-2630/18/8/083041.

Y. Zeng, P. Peng, Q.-J. Du, Y.-S. Wang, and B. Assouar, Journal of Applied Physics 128, 014901 (2020). https://doi.org/10.1063/1.5144177.

Z. Liu, K.-Q. Qin, and G.-L. Yu, Journal of Engineering Mechanics 146, 04020032 (2020). https://doi.org/10.1061/(ASCE)EM.1943-7889.0001752.

Y.-F. Wang, V. Laude, and Y.-S. Wang, Journal of Physics D: Applied Physics 47, 475502 (2014). https://doi.org/10.1088/0022-3727/47/47/475502.

Y.-F. Wang, Y.-S. Wang, and V. Laude, Physical Review B 92, 104110 (2015). https://doi.org/10.1103/PhysRevB.92.104110.

T.-T. Wu, Z.-G. Huang, and S. Lin, Physical review B 69, 094301 (2004). https://doi.org/10.1103/PhysRevB.69.094301.

S. Benchabane, A. Khelif, J.-Y. Rauch, L. Robert, and V. Laude, Physical Review E 73, 065601 (2006). https://doi.org/10.1103/PhysRevE.73.065601.

L. Cao, Y. Zhu, Y. Xu, S.-W. Fan, Z. Yang, and B. Assouar, Journal of the Mechanics and Physics of Solids, 104502 (2021). https://doi.org/10.1016/j.jmps.2021.104502.

Q. Gan, Y. Gao, K. Wagner, D. Vezenov, Y. J. Ding, and F. J. Bartoli, Proceedings of the National Academy of Sciences 108, $5169 \quad$ (2011). https://doi.org/10.1073/pnas.1014963108.

H. Hu, D. Ji, X. Zeng, K. Liu, and Q. Gan, Scientific reports 3, 1249 (2013). https://doi.org/10.1038/srep01249.

Y. Zeng, S.-Y. Zhang, H.-T. Zhou, Y.-F. Wang, L. Cao, Y. Zhu, Q.-J. Du, B. Assouar, and Y.S. Wang, arXiv preprint arXiv:2012.09928 (2020). arXiv:2012.09928.

J. Guo, J. Cao, Y. Xiao, H. Shen, and J. Wen, Physics Letters A 384, 126253 (2020). https://doi.org/10.1016/j.physleta.2020.126253.

A. O. Krushynska, M. Miniaci, F. Bosia, and N. M. Pugno, Extreme Mechanics Letters 12, 30 (2017). https://doi.org/10.1016/j.eml.2016.10.004. 